Supplement of The Cryosphere, 14, 4627-4651, 2020

https://doi.org/10.5194/tc-14-4627-2020-supplement

(c) Author(s) 2020. This work is distributed under

the Creative Commons Attribution 4.0 License.

(c) (1)

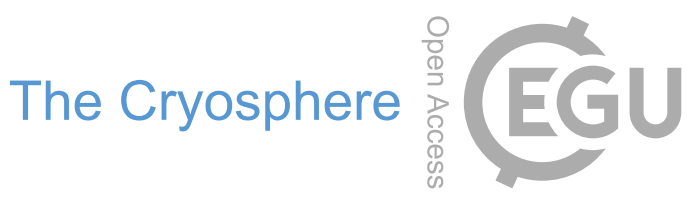

Supplement of

\title{
Numerical modelling of permafrost spring discharge and open-system pingo formation induced by basal permafrost aggradation
}

Mikkel Toft Hornum et al.

Correspondence to: Mikkel Toft Hornum (mth@ign.ku.dk)

The copyright of individual parts of the supplement might differ from the CC BY 4.0 License. 
The 1D heat transfer model (1DHT) consists of a MATLAB script (MathWorks®, 2019) tailored to simulate dynamics of ground temperatures and permafrost conditions. The model code is publicly available at DOI:10.5281/zenodo. 4240594.

Before the 1HDT model was applied in our investigation, it was validated through comparison with two analytical solutions.

5 In the following, we first describe the basis of the 1DHT model. Second, we present two different analytical solutions and show how they compare with numerical results as simulated by the 1DHT model.

\section{S1.1 Model code}

In the core of the 1DHT model is an explicit forward-difference time approximation of the one-dimensional conductive heat transfer (Eq. 1). For the interior cell, $i$, which is situated in a 1D grid in all of which the initial temperature, $T^{p}$, is known (Fig.

$10 \mathrm{~S} 1)$, this approximation states that at a new time, $(p+1)$,

$$
\begin{aligned}
& \frac{T_{i}^{p+1}-T_{i}^{p}}{\Delta t}=\alpha \frac{T_{i+1}^{p}-T_{i}^{p}+T_{i-1}^{p}}{\Delta x^{2}} \\
\Leftrightarrow \quad & T_{i}^{p+1}=\frac{\alpha \Delta t}{\Delta x^{2}}\left(T_{i+1}^{p}+T_{i-1}^{p}\right)+\left(1-2 \frac{\alpha \Delta t}{\Delta x^{2}}\right) T_{i}^{p}
\end{aligned}
$$

where $T_{i}^{p+1}$ is the temperature in cell $i$ at the time $(p+1), \Delta t$ is the time step, and $\Delta x$ is the distance to the center of the neighboring cells. The thermal diffusivity, $\alpha$, is calculated as defined by Eqs. (1), (3), and (4) in Sect. 4.1. Equation S1 is only

15 a stable numerical solution if the stability criterion is true (Bergman et al., 2011):

$$
\frac{\alpha \Delta t}{\Delta x^{2}} \leq \frac{1}{2} \Leftrightarrow \Delta t \leq \frac{\Delta x^{2}}{\alpha} \frac{1}{2}
$$

\begin{tabular}{|c|c|c|}
\hline$i-1$ & $i$ & $i+1$ \\
$i \Delta x$ & $i$ & \\
\hline
\end{tabular}

Figure S1 Conceptual representation of a 1D grid for which the 1DHT model simulates temperature changes (Eq. S1).

\section{S1.2 Neumann's solution}

20 In the case of phase change, conductive heat transfer becomes non-linear and the mathematical (Stefan) problems that arise may be of considerable difficulty (Carslaw and Jaeger, 1959). One of the exact solutions that deal with such a problem is the Neumann solution, which here is presented largely based on Mottaghy and Rath (2006). The presented version of the Neumann solution solves the 1D heat equation (Eq. 1), for a semi-infinite body, which consists of a single component and initially has the liquidus temperature. At the surface, the body is exposed to a negative temperature step change and the medium starts to change phase from liquid to solid. The position of the phase front, $Z$, is expressed as a function of time, $t$ :

$$
Z(t)=2 \cdot \gamma \cdot \sqrt{\alpha_{s o} \cdot t}
$$


where $\gamma$ is a parameter determined by

$$
\frac{\left(\exp \left(\left(\alpha_{s o}-\alpha_{l i}\right) \cdot \frac{\gamma^{2}}{\alpha_{l i}}\right) \cdot \operatorname{erfc}\left(\gamma \cdot \sqrt{\frac{\alpha_{S o}}{a_{l i}}}\right)\right)}{\operatorname{erf}(\gamma)}-\frac{\left(\left(T_{L}-T_{S}\right) \cdot k_{l i} \cdot \sqrt{\alpha_{s o}}\right)}{\left(T_{S}-T_{0}\right) \cdot k_{s o} \cdot \sqrt{\alpha_{l i}}}=0
$$

In both above equations, $\alpha$ 's are thermal diffusivities, $k$ 's are thermal conductivities, and subscripts $s o$ and $l i$ refer to the solid

30 or liquid state. $T_{L}$ and $T_{S}$ are the liquidus and solidus temperatures and $T_{0}$ is the surface temperature at $t>0$. The latent heat of fusion is incorporated in the expression of the thermal diffusivity of the liquid:

$$
\alpha_{l i}=\frac{k_{l i}}{\rho_{l i} \cdot c_{l i}+L \frac{-1}{T_{L}-T_{S}}}
$$

where $\rho_{l i}$ and $c_{l i}$ is the liquid's density and specific heat capacity, respectively.

To compare the 1DHT model with the exact Neumann's solution (Eq. S3), a situation which can be handled by both

35 methods, was defined: A body of pure water, initially at the liquidus temperature $T_{L}=0{ }^{\circ} \mathrm{C}$ is exposed to a surface temperature of $T_{0}=-4{ }^{\circ} \mathrm{C}$ for a period of $3000 \mathrm{yr}$. The thermal properties of ice and water are as listed Table 1 and the solidus temperature is $T_{S}=-2{ }^{\circ} \mathrm{C}$. In the following, these conditions will be referred to as Neumann conditions.

Solving Eq. S4 with the Neumann conditions, we found that $\gamma=0.0679$. Numerically, the 1DHT model simulated the temperature development in a 1D-grid containing 100 cells each with a length of 2 m. From the model simulation results,

40 the propagation of the freezing front was evaluated by interpolating $T=-2{ }^{\circ} \mathrm{C}$. The propagation of the freezing front, as calculated by Neumann's solution and evaluated from the 1DHT model simulation, is plotted on Fig. S2. The RMSE of the simulation results is 0.011 and show a relatively good model performance.

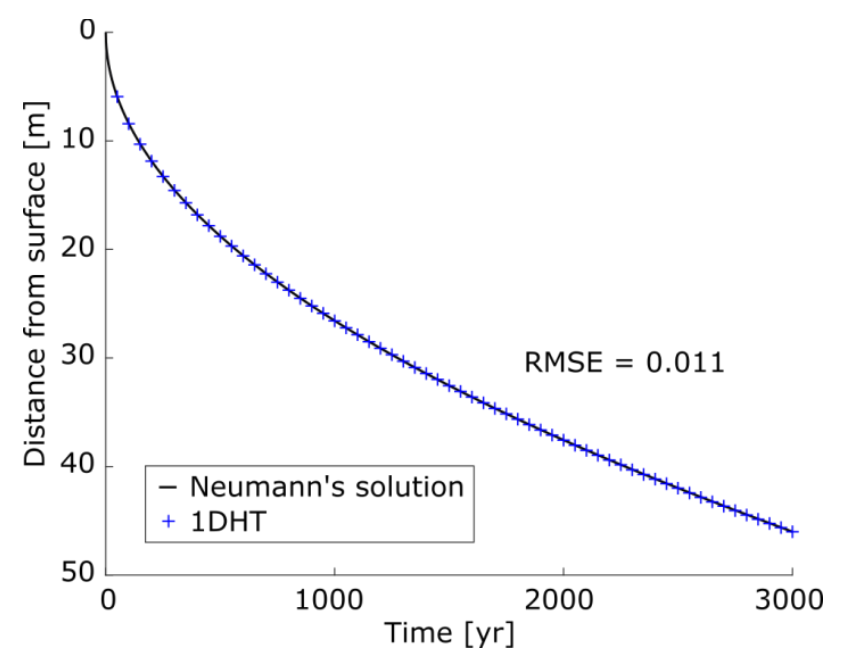

45 Figure S2 Freezing front depth with time in a body of water under Neumann conditions (see text) as calculated by the numerical 1DHT model and the analytical Neumann's solution. 


\section{S1.3 Step change in surface temperature (no latent heat effects)}

This analytical solution also assumes a negative temperature step change at the surface of a semi-infinite body: At the time $t=0$, the surface temperature is $T_{0 i}$, and at any later times $(t>0)$, it is $T_{0}$. As opposed to Neumann's solution, latent heat

50 effects are neglected, but the initial medium (e.g., ground) temperature can follow any (uniform) geothermal gradient and the medium may consist of several components. Following the step change in surface temperature $\left(\Delta T_{0}=T_{0}-T_{0 i}\right)$, the temperature change, $\Delta T$, is described as a function of time, $t$, and depth, $z$, by (Carslaw and Jaeger, 1959; Eppelbaum et al., 2014):

$$
\Delta T(z, t)=\Delta T_{0} \cdot \operatorname{erfc}\left(\frac{z}{2 \cdot \sqrt{\alpha_{e f f} \cdot t}}\right)
$$

55 where $\alpha_{e f f}$ is the effective thermal diffusivity. As with Neumann's solution, it was necessary to describe conditions, which this analytical solution can handle. The initial surface temperature was defined as $T_{0 i}=5^{\circ} \mathrm{C}$ and the ground temperature distribution followed a geothermal gradient of $0.025{ }^{\circ} \mathrm{C} \mathrm{m}^{-1}$. At times $t>0$, the surface temperature was defined as $T_{0}=0^{\circ} \mathrm{C}$ implying that $\Delta T_{0}=-5^{\circ} \mathrm{C}$. The medium had the geothermal properties of silty sand (Table 2 ) and a total porosity $\mathrm{n}=0.4$, which was fully saturated with water. This yields $\alpha_{e f f}=5.7 \mathrm{~m}^{2} \mathrm{yr}^{-1}$.

The numerical grid was set up as for the simulation of the Neumann conditions except for the temperature distribution, which followed the geothermal gradient as described above. The calculated ground temperature distribution from both the 1DHT model and the analytical solution at $t=1000 \mathrm{yr}$ is presented on Fig. S3. An excellent performance by the 1DHT model is indicated by a RMSE of $1.3 \cdot 10^{-5}$.

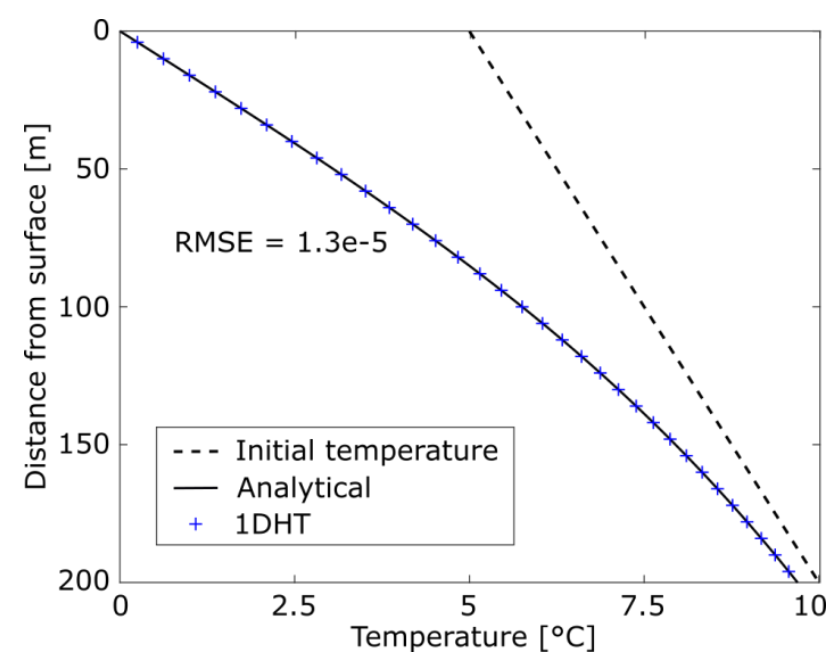

Figure S3 Comparison of the ground temperature distribution at $t=1000 \mathrm{yr}$ calculated analytically (Eq. S6) and as simulated by the 1DHT model. 
The vertical permeability, $\kappa_{v}$, of the sandstone-dominated Carolinefjellet and Helvetiafjellet formations were measured as part

70 of the Longyearbyen $\mathrm{CO}_{2}$ Laboratory Project (Olaussen et al., 2020, and references therein). The small-scale horizontal permeability, $\kappa_{h}$, for sandstones is typically a factor two higher than $\kappa_{v}$ (Domenico and Schwartz, 1998) and we converted the horizontal hydraulic conductivity, $K_{h}$, accordingly (Eq. 7). To tighten the $K_{h}$-range used in the groundwater model, we defined the realistic minimum, mean, and maximum values as the first, second and third quartiles of a statistical fit to the measured values. As illustrated on Fig. S4, three types of statistical distribution were tested; log-normal, Weibull and log-logistic (panels

75 a), b) and c), respectively). While the log-logistic fit showed the best correlation, all three fits predicted the same hydraulic conductivities at the quartiles.
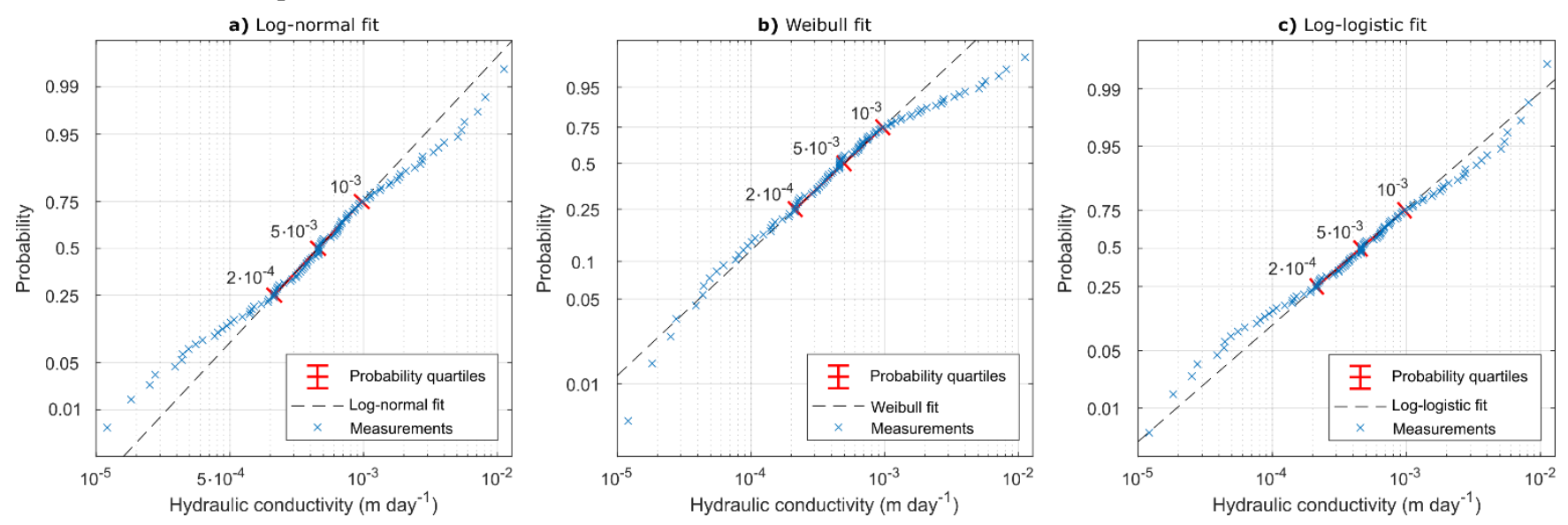

Figure S4 Log-normal (a), Weibull (b) and log-logistic (c) fits to the measured hydraulic conductivities of Carolinefjellet and Helvetiafjellet formations. Original data from the Longyearbyen $\mathrm{CO}_{2}$ Laboratory Project (Olaussen et al., 2020, and references therein). The log-logistic fit

80 shows the best correlation with the measured values and the values used in the groundwater model were thus defined by the quartiles predicted by this fit. Note that the two other fits both predict the same quartiles.

\section{S3 Major ions in Adventdalen pingo spring waters}

Hydrochemical data from 25 spring water samples from 2014 to 2017 presented by Hodson et al. (2020) and publicly available from DOI:10.5285/3d82fd3f-884b-47b6-b11c-6c96d66b950d give insights into the groundwater system in Adventdalen. As

85 illustrated in Fig. S6a, water samples from Lagoon (LP), Førstehytte (FHP), Innerhytte (IHP) and River Pingos (RP) reveal that all these springs share the same sodium-bicarbonate $\left(\mathrm{NaHCO}_{3}\right)$ water type. The only exception is four samples taken near River Pingo in 2017 of a magnesium-sulfate water type (Fig. S6b). These four samples were excluded from the discussion because they might not be associated with a pingo according to Hodson et al. (2020). 


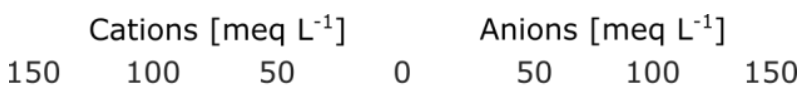

(a) All except River Pingo 2017

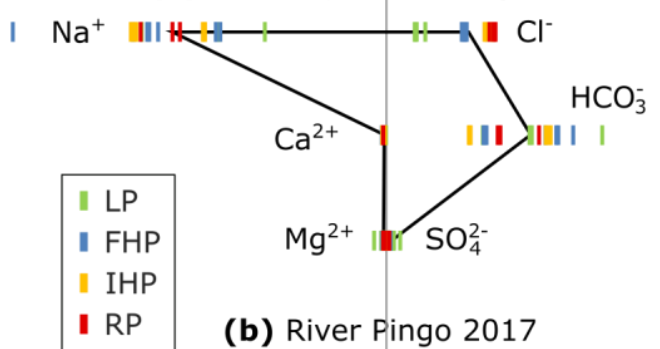

(b) River Pingo 2017

Figure S5 Stiff plots of major ion concentrations from pingo spring water samples in Adventdalen (data first presented in Hodson et al., 2020). The corners of the Stiff polygons represent mean concentrations.

\section{References}

Bergman, T. L., Lavine, A. S., Incropera, F. P. and Dewitt, D. P.: Fundamentals of Heat and Mass Transfer, 7th ed., John

95 Wiley \& Sons., 2011.

Carslaw, H. S. and Jaeger, J. C.: Conduction of heat in solids, 2nd ed., Clarendon Press., 1959.

Domenico, P. A. and Schwartz, F. W.: Physical and chemical hydrogeology, Wiley \& Sons., 1998.

Eppelbaum, L., Kutasov, I. and Pilchin, A.: Applied Geothermics, Lect. Notes Earth Syst. Sci., 757, doi:10.1007/978-3-64234023-9, 2014.

100 Hodson, A., Nowak, A., Hornum, M. T., Senger, K., Redeker, K. R., Christiansen, H. H., Jessen, S., Betlem, P., Thornton, S., Turchyn, A. V., Olaussen, S. and Marca, A.: Sub-permafrost methane seepage from open system pingos in Svalbard, Cryosph., doi:10.5194/tc-2020-11, 2020.

MathWorks®: MATLAB R2019b, [online] Available from: https://se.mathworks.com/products/matlab.html, 2019.

Mottaghy, D. and Rath, V.: Latent heat effects in subsurface heat transport modelling and their impact on palaeotemperature

105 reconstructions, Geophys. J. Int., 164(1), 236-245, doi:10.1111/j.1365-246X.2005.02843.x, 2006.

Olaussen, S., Senger, K., Braathen, A., Grundvåg, S.-A. and Mørk, A.: You learn as long as you drill; research synthesis from 
the Longyearbyen CO2 Laboratory, Svalbard, Norway, Nor. J. Geol., 99(2), 157-187, doi:10.17850/njg008, 2020. 